\title{
Buchbesprechung
}

\section{Ornamental Crops}

Johan Van Huylenbroeck (Editor), 2018. 887 Seiten, 187 Abbildungen, davon 166 in Farbe. Serie: Handbook of Plant Breeding, Vol. 11 (Editors-in-chief: Istvan Rajcan und Johann Vollmann), Springer International Publishing. Gebunden, Preis: 279,99 \$, eBook ISBN: 978-3-319-90698-0, Hardcover ISBN: 978-3-319-90697-3.

\author{
Buchbesprechung verfasst von: \\ Johannes Balas
}

Universität für Bodenkultur Wien, Department für Nutzpflanzenwissenschaften, Abteilung Gartenbau, Gregor-Mendel-Straße 33, 1180 Wien, Österreich, johannes.balas@boku.ac.at

Der in englischer Sprache erschienene Band wurde von Dr. Johan Van Huylenbroeck vom Flanders Research Institute for Agriculture, Fisheries and Food (ILVO, Melle, Belgien), einem der international renommiertesten und erfahrenen belgischen Zierpflanzenzüchter, herausgegeben. Das 887 Seiten umfassende Werk ist als Band 11 der Springer-Serie Handbook of Plant Breeding erschienen, die von Istvan Rajcan (University of Guelph, Kanada) und Johann Vollmann (Universität für Bodenkultur, Wien) editiert wird. Der Band ist in 29 Einzelkapitel gegliedert. Ein dem thematischen Umfang und der wirtschaftlichen Bedeutung der „globalisierten“ Wertschöpfungsketten im Zierpflanzenbau angemessenes, weltweites Konsortium von mehr als 80 Autorinnen und Autoren hat diese Beiträge geliefert. Die ersten neun Kapitel behandeln grundlegende Thematiken und bieten fundiert den aktuellen akademischen Wissenstand der Grundlagen im Bereich der Zierpflanzen (für den Innenraum wie auch die Freilandverwendung): etwa die Rolle der natürlichen Biodiversität, Erhaltung von Genotypen in Genbanken, die Genetik und genetische Modifikation der Blütenfarben (eines der wesentlichsten Merkmale des zierpflanzenbaulichen „Ästhetischen Wertes"), Züchtung zur Verbesserungen im Nachernte-Qualitätsmanagement von Schnittblumen (shelf-life; vase-life) oder die auch bei Zierpflanzen aktuell wichtiger werdende Züchtung auf Krankheitsresistenz. Zuchtmethodische und züchtungstechnische Abschnitte umfassen vor allem auch biotechnologisch relevante Felder: den Einsatz von Zellund Gewebekulturtechniken, die Einsatzmöglichkeiten von Polyploidie- und Mutationsinduktion sowie weitere molekulare Techniken, die zur markergestützten Selektion eingesetzt werden, aber auch der Identifikation von Sorten und damit dem Sortenschutz dienen.

Die Welt der Zierpflanzen lebt von der großen Diversität an Arten und Sorten in Produktion und Verwendung - seriöse Schätzungen gehen von bis zu 5000 kultivierten Arten weltweit aus. Daher überrascht es nicht, dass ganze zwanzig Buchkapitel den individuellen Gattungen, Artengruppen oder Arten gewidmet sind. Der Aufbau dieser Kapitel ist nicht stringent einheitlich, folgt aber einem ähnlichen Muster: Einführung in die Art bzw. Gruppe, Taxonomie, Geschichte der Sorten und deren Entwicklung, Blütenbzw. Befruchtungsbiologie, Genetik, Züchtungstechniken, spezifische Zuchtziele und vielfach Ausblicke auf künftig mögliche bzw. angestrebte Entwicklungen. Kapitel, wie jenes über die Züchtung der Rosen, der wirtschaftlich wie kulturell nach wie vor wichtigsten Zierpflanzengruppe, sind mit 50 Seiten sehr umfangreich ausgefallen, tragen aber den verschiedenen Nutzungsbereichen von Gartenrosen über Topfrosen bis Schnittrosen und von der Genomforschung bis zur praktischen Züchtung Rechnung. Auch weiteren Hauptkulturen wie Tulpen, Nelken, aber 
auch beispielsweise Alstroemerien, Chrysanthemen, Lilien und Flammendes Käthchen wird „Referenz erwiesen“. Es findet sich viel Interessantes von A bis Z ! Hervorgehoben seien exemplarisch die (an sich essbaren) Zierpaprika und -chili, aber auch Azaleen, Fuchsien, Gerbera, Phalaenopsis-Orchideen, Primeln und Rhododendren. Eine Besonderheit stellt wohl das beinahe exotisch zu nennende, abschließende Kapitel zur Züchtung der verholzenden Arten der gemäßigten Klimazone dar.

Das Handbuch wendet sich einerseits an akademisch Forschende, Lehrende und Studierende, aber andererseits sehr wohl auch an kommerziell orientierte Pflanzenzüchter und Genetiker. Es bietet sich jedoch auch als eine reichhaltige Quelle an Informationen für die große Zahl Interessierter an den diversen Aspekten des Zierpflanzenbaus an. Alle Gruppen finden hier eine tatsächlich einmalige Zusam- menstellung einer respektablen Fülle an gut gegliederter und aufbereiteter Information vor. Jedes einzelne Kapitel bietet abschließend eine große Anzahl an Literaturquellen für eine weitere Vertiefung an.

Damit füllt dieses Kompendium eine bereits viel zu lange bestehende Lücke in der wissenschaftlichen Literatur zu den Kulturgruppen selbst und der Züchtung der Zierpflanzen im Speziellen. Es zeigt auch auf eindrucksvolle Weise, dass trotz der Schnelllebigkeit in der Academia und allzu oft auf den kurzfristigen Erfolg ausgerichtetes Arbeiten, exzellente Publikationstätigkeit in Buchform möglich und vor allem sinnvoll ist. Ein Werk mit durchgängig hohem Niveau und Aktualität, dem ein erfolgreicher Einsatz in Lernen, Lehre und Forschung ehrlich zu wünschen ist. 